\title{
Protein quality control machinery in intracellular protozoan parasites: hopes and challenges for therapeutic targeting
}

\author{
Mohammad Anas $^{1} \cdot$ Varsha Kumari $^{1} \cdot$ Niharika Gupta $^{1} \cdot$ Anuradha Dube $^{1} \cdot$ Niti Kumar $^{2}$ (D
}

Received: 7 March 2019 /Revised: 8 June 2019 / Accepted: 10 June 2019 /Published online: 21 June 2019

(C) Cell Stress Society International 2019

\begin{abstract}
Intracellular protozoan parasites have evolved an efficient protein quality control (PQC) network comprising protein folding and degradation machineries that protect the parasite's proteome from environmental perturbations and threats posed by host immune surveillance. Interestingly, the components of PQC machinery in parasites have acquired sequence insertions which may provide additional interaction interfaces and diversify the repertoire of their biological roles. However, the auxiliary functions of PQC machinery remain poorly explored in parasite. A comprehensive understanding of this critical machinery may help to identify robust biological targets for new drugs against acute or latent and drug-resistant infections. Here, we review the dynamic roles of PQC machinery in creating a safe haven for parasite survival in hostile environments, serving as a metabolic sensor to trigger transformation into phenotypically distinct stages, acting as a lynchpin for trafficking of parasite cargo across host membrane for immune evasion and serving as an evolutionary capacitor to buffer mutations and drug-induced proteotoxicity. Versatile roles of PQC machinery open avenues for exploration of new drug targets for anti-parasitic intervention and design of strategies for identification of potential biomarkers for point-of-care diagnosis.
\end{abstract}

Keywords Chaperones $\cdot$ HSP $\cdot$ Protein quality control $\cdot$ Parasite $\cdot$ Plasmodium, Leishmania, Toxoplasma, Trypanosoma

$\begin{array}{ll}\text { Abbreviations } \\ \text { PQC } & \text { Protein quality control } \\ \text { HSPs } & \text { Heat shock proteins } \\ \text { sHSP } & \text { Small heat shock proteins } \\ \text { RBC } & \text { Red blood cells } \\ \text { PV } & \text { Parasitophorous vacuole } \\ \text { GRASP } & \text { Golgi reassembly stacking protein } \\ \text { RESA } & \text { Ring-infected erythrocyte surface antigen }\end{array}$

\section{Introduction}

Protozoan parasites include a diverse class of extracellular and intracellular eukaryotic microbes that cause fatal infections in humans and animals (Aikawa 2012;

Niti Kumar

niti.kumar@cdri.res.in

1 Department of Parasitology, CSIR-Central Drug Research Institute, Lucknow, Uttar Pradesh 226031, India

2 Academy of Scientific and Innovative Research (AcSIR), Delhi, India
Walochnik et al. 2017). The extracellular protozoan parasites are free living and are transmitted to human host mainly through contaminated food and water (Entamoeba histolytica, Giardia intestinalis), or through contact with infected mucosal membranes (Trichomonas vaginalis), and in some cases through an invertebrate vector (Trypanosoma brucei) (Piña-Vázquez et al. 2012), whereas intracellular parasitic infections involve zoonotic (toxoplasmosis, diarrhea) or vector-borne transmission (malaria, black fever, Chagas disease) (Bates 2018; Dubey 2014; Messenger et al. 2015; Phillips et al. 2017; Ryan et al. 2014). The critical features of these intracellular parasites are (i) breach through different anatomical barriers in host and/or vector, (ii) transformation into morphologically and functionally distinct stages to survive/multiply in hostile intracellular environments, and (iii) successful transmission (through vector or intermediate host) (Walker et al. 2014). Among the intracellular protozoan parasites, Plasmodium, Toxoplasma, Leishmania, and Trypanosoma exhibit a disproportionate global disease burden with enormous clinical variations ranging from asymptomatic to lifethreatening conditions suggesting a geographic mosaic 
in host-parasite coevolution. Further, the emerging drug resistance and limited window of opportunity with current front-line drug regimens for acute and latent infections (Flegr et al. 2014; WHO 2014) have increased the need for more intensive efforts to understand parasite biology, to identify robust biological targets, and to design pharmacologically active scaffolds.

In this review, we have focused on representative intracellular parasites from apicomplexans (Plasmodium, Toxoplasma) and kinetoplastids (Leishmania, Trypanosoma). These intracellular protozoan parasites have undergone unique adaptations to support their complex life cycle in multiple hosts, such as (i) reside within the protected niche of the membrane-bound vacuole inside the host cell, (ii) acquire unique organelles like a relict plastid called apicoplast (e.g., Plasmodium, Toxoplasma) and kinetoplast DNA network harboring mitochondrion (e.g., Leishmania, Trypanosoma) to perform critical metabolic functions, and (iii) evolve unique strategies to ensure successful entry and exit from the infected cell. A
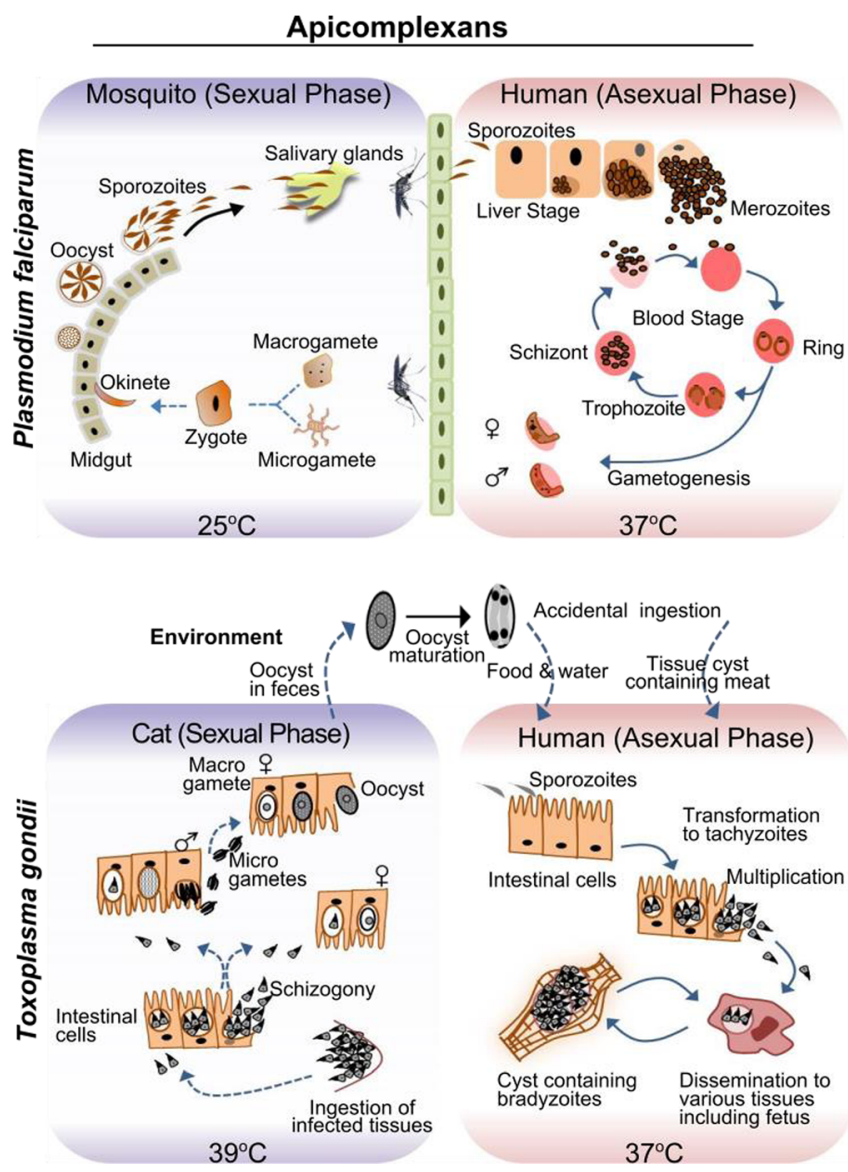

Fig. 1 Comparing the complex digenetic life cycle of representative intracellular parasites (P. falciparum, T. gondii, L. major, and T. cruzi). These parasites encounter frequent environmental fluctuations (temperature, $\mathrm{pH}$, nutrients) and undergo multiple phenotypic transitions during their life cycle. Depending upon environmental glance into their complex life cycle reveals that these intracellular parasites encounter frequent fluctuations in temperature, $\mathrm{pH}$, nutrients, and oxygen as they home in different tissues and transit through phenotypically unique stages (Fig. 1, Box 1). For instance, in Plasmodium, the asexual life cycle begins with injection of sporozoites (motile parasites) into a mammalian host during a blood meal of the female Anopheles mosquito and the sexual cycle takes place in the mosquito vector. In the case of Toxoplasma, the parasite undergoes the asexual cycle in human (an intermediate host) and the sexual cycle is completed in cat (definitive host). In Leishmania and Trypanosoma, however, the parasite undergoes asexual reproduction in both human and its respective vector. Recent developments have shown that both these parasites may also undergo genetic exchange (albeit at low frequencies) during their development in vector (Akopyants et al. 2009; Ocaña-Mayorga et al. 2010).

Box 1 Life cycle of representative intracellular protozoan parasites
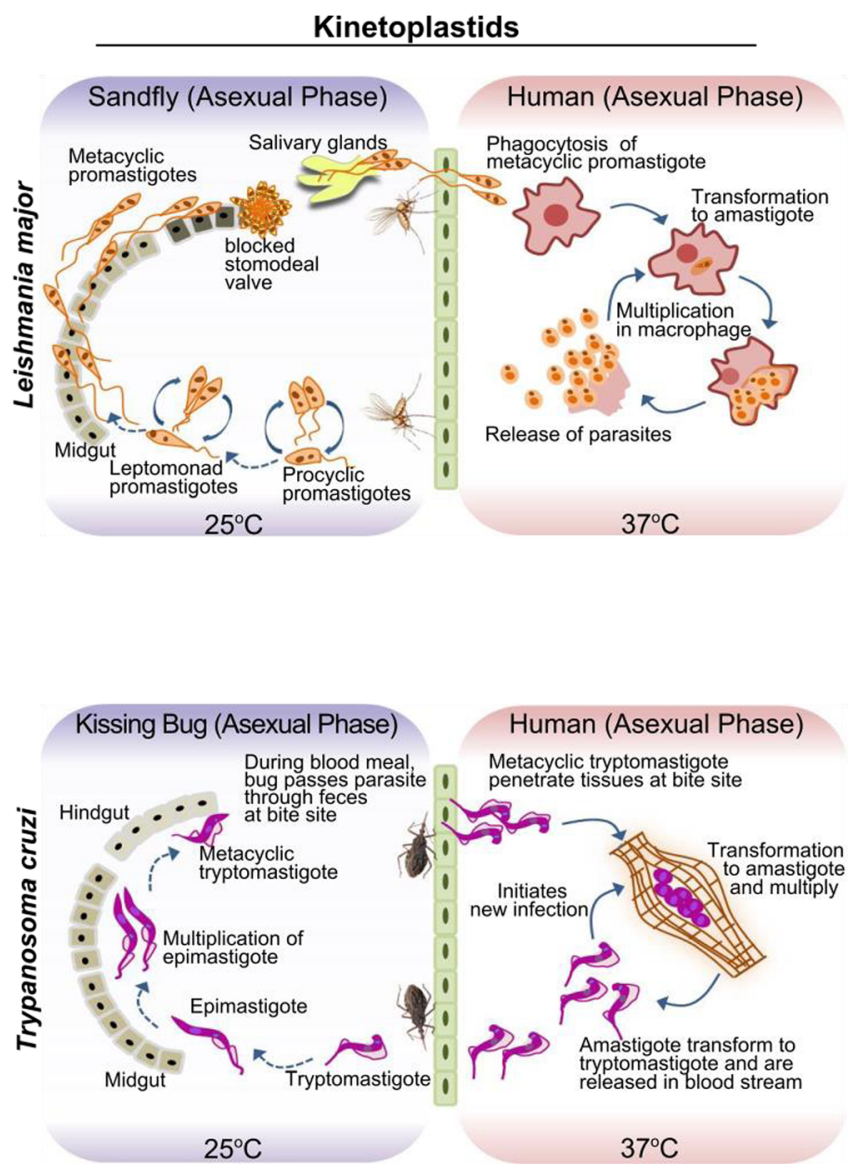

factors, the parasite makes critical decisions for proliferation and transmission. Plasmodium spp. and Toxoplasma are spore-forming protists harboring a relict plastid (apicoplast) and apical complex. Leishmania spp. and Trypanosoma spp. are flagellated protists that have unusual kinetoplast-DNA harboring mitochondrion 
Plasmodium spp. cause malaria in humans and animals. Infection begins with the injection of sporozoites (50 per bite) and anti-coagulating saliva during the blood meal of female mosquito (Anopheles spp.). Parasite glides through skin and through lymphatic or blood vessels invading into liver cells (30-60 min after mosquito bite). The surface proteins of parasite bind to the liver heparin sulfate proteoglycans and invade into the liver cell to multiply through schizogony (7-10 days) to release merozoites $(>10,000)$ into bloodstream. In case of $P$. vivax and $P$. ovale, the parasite enters a dormant state which causes relapse fever. The released merozoites invade into RBCs by binding to RBC surface receptors. The invaded parasite undergoes multiple transitions to ring, trophozoite, schizont, and merozoite stage ( $24 \mathrm{~h}$ in rodent and $48 \mathrm{~h}$ in human). The infected RBCs lyse to release merozoites (8-30 depending on Plasmodium spp.) and endotoxins. Fever spikes correlate with RBC lysis. Environmental and host factors trigger gametogenesis during the asexual phase in RBCs. The gametocytes are taken up by the mosquito during its blood meal. In the midgut, the gametes fuse to form zgyote that differentiates into motile ookinete. The ookinete crosses midgut and forms matured oocyst, wherein sporozoites develop. The released sporozoites migrate to colonize in salivary glands and the entire sexual phase in mosquito which takes 11-14 days before the mosquito is ready for its next transmission cycle into the human host.

Toxoplasma causes toxoplasmosis in human and animals. Humans are accidental host that get infected by consummation of oocyst-contaminated food and water or meat contaminated with tissue cyst. Sporulated oocyst releases sporozoites $(1-4 \mathrm{~h})$ to invade into intestinal cells and bradyzoites in tissue cyst transform into actively dividing tachyzoites $(18 \mathrm{~h})$ which further invade into other cells (2 days) and also crosses the placenta to infect the fetus. Depending upon host environmental factors, the parasite transforms into slowly dividing bradyzoites to form tissue cyst ( 7 days). As the environmental conditions become favorable, bradyzoite converts into tachyzoites $(18 \mathrm{~h})$. Feline host is the definitive host in which sexual cycle begins with ingestion of tissue cyst-infected meat (Toxoplasma-infected rodents or birds). The parasite infects the intestinal tissues and undergoes schizogony. The released tachyzoites infect new tissues and undergo gametogenesis to form micro and macro gametes that fuse to form an oocyst. The oocyst is released in cat's feces and undergoes maturation in an external environment. The time span for excretion of oocyst in feces is 3-10 days after ingestion of bradyzoite-infected tissues.

Leishmaniasis is caused by Leishmania spp. resulting in cutaneous, mucocutaneous, or visceral infections in humans and animals. Female sandfly (Phlebotomus spp.) bite releases motile metacyclic promastigotes which enter macrophages through phagocytosis and undergoes transformation into non-motile amastigotes (3-4 h) and multiplication of amastigotes (3-4 days). Macrophage lysis releases amastigote to initiate fresh infection in various tissues (skin, liver, spleen, bone marrow). For transmission, the parasite is taken by sandfly during bite $\left(10^{3}-10^{6}\right.$ parasites per fly) and it undergoes transformation into different stages in sandfly midgut (14-20 days). The parasite detaches from the midgut and migrates to the thoracic midgut, where due to secretion of the promastigote secretory gel (PSG), the stomodeal valve is blocked. The blocked sandflies are unable to complete a full blood meal and thus attempt to feed with greater frequency, increasing the chance of parasite transmission. This forces sandfly to regurgitate PSG before it can blood feed, thereby depositing both PSG \& infective metacyclic promastigote in the skin of host.

Trypanosoma spp. includes both extracellular (T. brucei causes sleeping sickness) and intracellular protozoan parasite (T. cruzi causes Chagas disease). T. brucei is transmitted during blood meal of infected Tsetse fly (Glossina spp.) which injects the metacyclic stage of trypomastigotes, and they develop into long and slender forms in the vertebrate host, while, in T. cruzi infections, the parasite is released in feces of kissing bug (Triatoma spp.) at the bite or wound site. Flagellated metacyclic trypomastigotes are $20 \mu \mathrm{m}$ long with a centrally located nucleus and posteriorly located kinetoplastid. The motile parasites penetrate the tissues, transform into non-motile amastigote ( $2-4 \mu \mathrm{m}$ diameter), and multiply for 3-5 days. Depending on host factors (nutritional and environmental), the amastigote transforms back to trypomastigote and is released in the blood stream to invade new cells. The parasite is taken by the kissing bug during blood meal; it undergoes numerous transformations, multiplies through longitudinal binary fission, and migrates from midgut to hindgut of the kissing bug. After 8-10 days, the parasite is released as tryptomastigote in feces during the blood meal by the kissing bug.

Recent evidences suggest existence of sexual reproduction in Leishmania and Trypanosoma (albeit at low frequencies of genetic exchange) during their development in vector (Akopyants et al. 2009; Ocaña-Mayorga et al. 2010).

For a successful infection, intracellular parasites have evolved unique approaches for invasion and exit from a host cell. For entry into a host cell, parasites engage secretory organelles at the apical pole to support motility-driven invasion (Plasmodium, Toxoplasma) (Frénal et al. 2017), host cellmediated phagocytosis (Leishmania) (Lodge and Descoteaux 2008), calcium-dependent signaling, and PI3 kinase-dependent recruitment of lysosomes at the wound site (Trypanosoma) (Cortez et al. 2016). Egress strategies include activation of parasite proteases and disruption of host cytoskeleton to destabilize plasma membrane (Plasmodium) (Burda et al. 2017; Hale et al. 2017), apoptosis (Leishmania) (Real et al. 2014), inhibition of the Rho kinase signaling pathway-mediated cytoskeleton remodeling (Trypanosoma) (Mott et al. 2009), calcium-dependent stimulation of microneme proteins (containing proteolytic enzymes), and secretion (Toxoplasma) (McCoy et al. 2012). The parasite also ensures successful transmission for a new infection cycle, (i) through salivary glands of vector (Plasmodium spp., Leishmania spp.), (ii) through excretion from the anus by vector at wound site (Trypanosoma cruzi), or (iii) through ingestion of contaminated food and water (Toxoplasma gondii). These parasites also manipulate the vector to increase their transmission rates by inducing changes in vector physiology leading to higher frequency and longer duration of contact between vector and host. Parasites also cause changes in odor or behavior of infected host to enhance their transmission to healthy hosts (Ferguson and Read 2004; O'Shea et al. 2002; Vyas 2015).

To sustain their complex life cycle in distant hosts, these intracellular parasites have evolved versatile proteomes harboring proteins with low-complexity/disordered regions allowing them to adopt dynamic conformations to execute diverse functions (Battistuzzi et al. 2016; Muralidharan and 
Goldberg 2013). However, this structural-functional advantage of proteome encoding disordered proteins is accompanied by an intrinsic vulnerability to protein misfolding and aggregation. How then is the parasite able to keep its metastable aggregation-prone proteome in a functional state against the environmental challenges encountered during its life cycle? One plausible explanation is that they may have evolved efficient protein quality control (PQC) machinery that limits protein misfolding and efficiently removes the aggregated species. The PQC network has interconnected protein folding and degradation machineries that coordinate the maintenance of protein homeostasis (Klaips et al. 2018; Powers and Balch 2013). These components have been extensively studied in human host, but their parasitic orthologs remain poorly explored (Shonhai et al. 2011). Proteome-wide phylogenetic analysis revealed that components of PQC machinery have significantly diverged in parasites, suggesting the acquisition of moonlighting roles for survival fitness (Bhartiya et al. 2015). Until recently, research efforts were focused on the canonical roles of the parasite's protein folding or degradation machinery without any discussion of their potential noncanonical roles. In this review, we highlight the versatile functions of components of PQC machinery in intracellular protozoan parasites, Plasmodium, Toxoplasma, Leishmania, and Trypanosoma, as these parasites have a complex digenetic life cycle, survive in hostile cellular environments, and undergo developmental processes in evolutionary distant organisms.

\section{Potential dynamic roles of protein quality control machinery in intracellular protozoan parasites}

Various components of PQC machinery participate in co- and post-translational folding, processing, targeting, assembly, and degradation of nascent polypeptides (Craig and Marszalek 2017; Dikic 2017; Kim and Hwang 2013; Klaips et al. 2018; Mogk et al. 2019; Schopf et al. 2017; Yebenes et al. 2011). The PQC machinery in intracellular protozoan parasites has few conserved and many diverged components (Fig. 2) that exhibit considerable species-specific variation within a phylum. Protein blast analysis has revealed that some of these components have sequence insertions of variable length and complexity (containing repeats of single amino acid or short amino acid motifs) (Bhartiya et al. 2015; Chaudhry et al. 2018; Singh et al. 2004). These sequence insertions provide additional interaction interfaces that may facilitate execution of multifarious roles in survival, multiplication, and transmission of the parasite. Research efforts for understanding the parasite's PQC machinery are mainly limited to canonical roles investigated in model organisms (Pavithra et al. 2007;

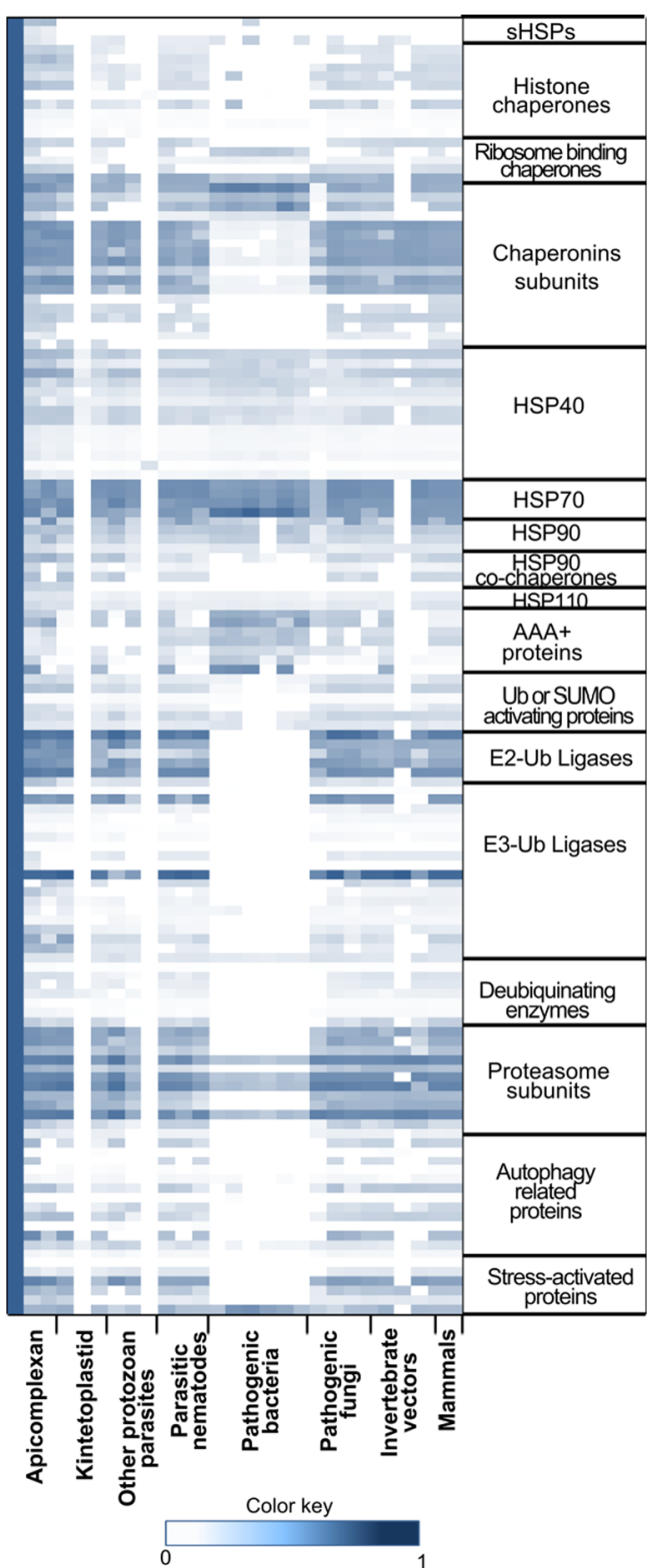

Fig. 2 Heat map representing the evolutionary divergence of components of protein quality control machinery across various proteomes (across $x$ axis, left to right). In this heat map, for each query of the $P$. falciparum protein, a normalized similarity score of respective ortholog in the queried organism is represented in the scale of 0 (white, low similarity) to 1 (blue, high similarity). For instance, HSP70 shows high conservation across proteomes, whereas its co-chaperone HSP40 displays enormous divergence (across proteomes, $x$-axis) (data is taken from Bhartiya et al. 2015)

Pesce et al. 2014; Requena et al. 2015; Shonhai et al. 2011; Vonlaufen et al. 2008). However, few reports also suggest their potential role in genome-epigenome maintenance, metabolic processes, differentiation, protein export, and immune evasion. The dynamic functions of the parasite's PQC machinery are summarized in Table 1, and their participation in various cellular processes are discussed in subsequent sections. 
Table 1 Moonlighting roles of components of protein quality control machinery in intracellular protozoan parasites

\begin{tabular}{|c|c|c|}
\hline Protein family & Functions & Ref. \\
\hline sHSPs & $\begin{array}{l}\text { Involved in cellular adhesion and migration } \\
\text { Also promotes thermotolerance and intracellular survival } \\
\text { Stage-specific expression may influence cellular decision of } \\
\text { proliferation and differentiation. }\end{array}$ & $\begin{array}{l}\text { Montagna et al. 2012; Hombach et al. } \\
\text { 2014; (Perez-Morales and Espinoza } 2015\end{array}$ \\
\hline Histone chaperones & $\begin{array}{l}\text { Folding, assembly, nuclear import, and degradation of histones } \\
\text { DNA unwinding during replication, recombination, and repair } \\
\text { Helps in recruitment of epigenetic machinery on DNA } \\
\text { Survival and fertility of gametes }\end{array}$ & $\begin{array}{l}\text { Navadgi et al. 2006; Laurentino et al. 2011; } \\
\text { Scher et al. 2012; Alsford and Horn } 2012\end{array}$ \\
\hline HSP40 & $\begin{array}{l}\text { Potential roles in parasite protein trafficking and export of virulence } \\
\text { factors }\end{array}$ & $\begin{array}{l}\text { Botha et al. 2007; Kulzer et al. 2010; Pei } \\
\text { et al. } 2007\end{array}$ \\
\hline Chaperonin & Suggested to play a role in protein trafficking and drug resistance & Mok et al. 2015 \\
\hline HSP70 superfamily & $\begin{array}{l}\text { Involved in nuclear and organellar genome maintenance, }[\mathrm{Fe}-\mathrm{S}] \text { cluster } \\
\text { biogenesis and assembly, modulation of immune response, } \\
\text { involved in trafficking of virulence factors, antigen presentation }\end{array}$ & $\begin{array}{l}\text { Kumar et al. 2010; Týč et al. 2015; Lukes } \\
\text { and Basu 2015; Dobbin et al. } 2002\end{array}$ \\
\hline HSP90 & $\begin{array}{l}\text { Interaction with epigenetic modifiers, differentiation, probable role } \\
\text { in drug resistance } \\
\text { Maintenance of telomere homeostasis and serving as an evolutionary } \\
\text { capacitor }\end{array}$ & $\begin{array}{l}\text { Pallavi et al. 2010; Echeverria et al. 2010; } \\
\text { Kaur et al. 2017; }\end{array}$ \\
\hline $\mathrm{AAA}+$ proteins & $\begin{array}{l}\text { Includes Clp proteases and HSP100 disaggregase machinery } \\
\text { Also implicated in parasite survival, virulence, and protein export }\end{array}$ & $\begin{array}{l}\text { Hübel et al. 1997; de Koning-Ward et al. } \\
2009\end{array}$ \\
\hline $\begin{array}{l}\text { Ubiquitin proteasome } \\
\text { system }\end{array}$ & $\begin{array}{l}\text { Dampening of host immune response, influences host signaling through } \\
\text { post-translational modification, and is also associated with drug resistance. }\end{array}$ & $\begin{array}{l}\text { Loureiro and Ploegh 2006; Maculins et al. } \\
\text { 2016; Haldar et al. } 2018\end{array}$ \\
\hline Autophagy related proteins & Involved in organelle biogenesis & Bansal et al. 2017; Walczak et al. 2018 \\
\hline Stress-activated proteins & $\begin{array}{l}\text { Generally perform metabolic functions, but during exposure to stress, they } \\
\text { also exhibit ATP-independent chaperone activity and undergo conforma- } \\
\text { tional rearrangements }\end{array}$ & Teixeira et al. 2019 \\
\hline
\end{tabular}

\# The role of HSP90 in telomere homeostasis and as an evolutionary capacitor is based on literature evidences in model organisms (DeZwaan and Freeman 2010; Cowen and Lindquist 2005; Rutherford and Lindquist 1998)

\section{Chaperoning the genome and epigenome}

Intracellular protozoan parasites exhibit finely regulated gene expression to adapt to frequent environmental perturbations encountered during their life cycle. It will be interesting to understand whether the parasite's PQC machinery plays important roles in modulation of the genome and epigenome landscape. In eukaryotic model organisms, numerous histone chaperones aprataxin PNK-like factor (APLF), antisilencing function protein 1 (ASF1), chromatin assembly factor 1 (CAF1), histone regulation A (HIRA), facilitates chromatin transcription (FACT), death domain-associated protein 6 (DAXX), minichromosome maintenance protein 2 (MCM2), nucleolin, p400, nucleosome assembly proteins (Naps), and patient SE translocation (SET) have been suggested to play essential roles in folding, assembly, post-translational modification, nuclear import, and turnover of histones (Hammond et al. 2017). These histone chaperones lack the canonical ATPase domain and require numerous accessory proteins to coordinate with DNA replication, recombination, repair, and transcription machineries. However, research on histone chaperones in intracellular parasites is quite limited. Plasmodium NapL and NapS exhibit variable affinities to histones and are differentially localized in the cytosol and nucleus. NapL facilitates the formation of the histone-NapS complex which is imported into the nucleus for chromatin assembly (Navadgi et al. 2006). Another histone chaperone, plasmodial FACT is suggested to be essential for survival and fertility of male gametes (Laurentino et al. 2011). Whereas plasmodial CAF1 is suggested to be important for maintenance of heterochromatin state in telomeric and sub-telomeric regions (Gupta et al. 2018), Trypanosoma ASF1 is thought to play important roles in DNA repair (Scher et al. 2012) and antigenic variation gene expression in parasites (Alsford and Horn 2012).

Apart from histone chaperones, the classical HSP70-40 and HSP90 chaperones have been shown to play key roles in nuclear and organellar genome maintenance. For instance, plasmodial DNAJ interacts with the replication ori of $35 \mathrm{~kb}$ circular DNA in apicoplast, presumably playing a role in replication 
and repair of the apicoplast genome (Kumar et al. 2010). Similarly in Trypanosoma, mitochondrial HSPs (mtHSP70/mtHSP40) and their nucleotide exchange factor (Mge1) are indispensable for the maintenance of mini $(0.5-2.5 \mathrm{~kb})$ and maxicircle (20$50 \mathrm{~kb}$ ) kinetoplastid DNA (Týč et al. 2015). Studies in model organisms have shown the role of HSP90co-chaperones in maintenance of telomere dynamics by direct interaction with telomerase and telomere binding proteins (DeZwaan and Freeman 2010). HSP90 is also proposed to chaperone the epigenome through its interaction with various epigenetic modifiers (Sawarkar and Paro 2013). In fact, the cross-talk with epigenetic modifiers modulates HSP90 functions by regulating its post-translational modifications (acetylation and deacetylation). A study in Plasmodium and Trypanosoma has shown that PanHDAC inhibitors cause hyperacetylation of parasite HSP90 which interferes with its protein folding activities critical for parasite survival (Pallavi et al. 2010).

\section{Tipping the balance between growth and differentiation}

Cellular proliferation, differentiation, and quiescence are determined by interaction of the parasite with hostile environments both in host and vector. Intracellular protozoan parasites (Plasmodium, Toxoplasma, Leishmania, Trypanosoma) reside and multiply in a membrane-bound vacuole called the parasitophorous vacuole (PV). The PV provides a safe haven to grow, differentiate, and undergo the metabolic rewiring upon exposure to environmental fluctuations (Liévin-Le Moal and Loiseau 2016; Spielmann et al. 2012). The PQC machinery may play an important role in nutrient uptake from the host cell and maintenance of metabolic homeostasis to support parasite growth. Yeast two-hybrid data suggests that $P$. falciparum HSP70/ HSP90-organizing protein (HOP) interacts with falcipain-2, a plasmodial cysteine protease involved in hemoglobin digestion to maintain amino acid pool for parasite growth (LaCount et al. 2005). HOP is a co-chaperone of both HSP70 and HSP90 (Alvira et al. 2014) that facilitates the transfer of client substrates (for example, critical parasite proteases) from HSP70 to HSP90 for chaperoning them into the functional structure. Further, organellar HSP70 and its cochaperones play a critical role in $[\mathrm{Fe}-\mathrm{S}]$ cluster biogenesis and assembly which are critical for the parasite's metabolic processes (Lukes and Basu 2015). Recently, components of autophagy (ATG8, ATG18) have been shown to be essential for apicoplast biogenesis in apicomplexan parasites (Bansal et al. 2017;
Walczak et al. 2018). Although both ATG8 and AT18 have autophagy-dependent functions, their role in apicoplast biogenesis is clearly autophagy-independent. Parasites also harbor stress organelles such as acidocalcisomes and glycosomes to support their survival during various environmental perturbations. Acidocalcisomes are acidic organelles with high concentrations of phosphorus ( $\mathrm{Pi}, \mathrm{PPi}$, polyP) complexed with calcium and other mono/divalent cations to protect the parasite from osmotic shock, and this organelle is also thought to play a role in autophagy ( $\mathrm{Li}$ and He 2014; Miranda et al. 2008). Glycosomes mainly possess glycolytic enzymes along with proteins involved in the pentose-phosphate pathway, nucleotide biosynthesis, and antioxidant enzymes (e.g., peroxiredoxins) (Szoor et al. 2014). Some antioxidant enzymes also exhibit stress-induced chaperoning activity, and hence, they are classified as stressactivated chaperones. These proteins have been mainly investigated in model organisms and have been poorly explored in parasites. These proteins undergo enormous structural rearrangements to adopt oligomeric structures to protect the unfolded polypeptide during stress (Voth and Jakob 2017). In parasites, the role of stress-activated chaperones is only reported for mitochondrial peroxiredoxins of Leishmania. Recent cryo-EM revealed that this decameric enzyme undergoes temperature-dependent structural rearrangements to expose chaperone-client binding sites that are buried in the peroxidase-active protein (Teixeira et al. 2019).

Cellular stress can cause metabolic reprogramming (a switch between oxidative phosphorylation and glycolysis) and differentiation (slow to actively dividing stages) (Salcedo-Sora et al. 2014) which is also coupled with stress-induced upregulation and/or differential localization of parasite HSPs. For instance, differential localization of Toxoplasma HSP90 and its co-chaperone p23 was observed upon transformation of rapidly dividing tachyzoites to the slowly growing bradyzoites (Echeverria et al. 2010). In Trypanosoma, HSP70/HSP90 co-chaperone (HOP) and HSP70 colocalization in cytoplasmic stress granules during nutrient-depleted condition is thought to be associated with selective storage of transcripts involved in differentiation (Schmidt et al. 2018). The probable role of the parasite's PQC machinery in stress granules can be the reinitiation of translation of stalled nascent polypeptides, facilitating their folding and proteinprotein interactions to rapidly regulate bioenergetics and differentiation events in response to stress. In Leishmania, changes in expression of HSPs (HSP90, HSP70, HSP60, HSP100, HSP40s, HSP23, SGT, 
STI1, TCP20), and components of autophagy machinery (ATG3, ATG4) were observed upon conversion of flagellated promastigote (extracellular asexual form) to non-motile amastigote (intracellular asexual form) (Besteiro et al. 2006; Morales et al. 2010; Requena et al. 2015). Further, Leishmania HSP100 (Clp protease), an ATP-dependent unfolding machinery, is proposed to be important for virulence and intracellular survival in a mammalian host (Hübel et al. 1997). Likewise, sHSPs are suggested to play a role in thermotolerance (in Leishmania), cellular adhesion, and migration (in Plasmodium) (Hombach et al. 2014; Montagna et al. 2012). Further, stage-specific expression of sHSPs may influence the proliferation and differentiation potential of parasites (Perez-Morales and Espinoza 2015). sHSPs may act as an adaptor for protein-protein interaction and in regulating subcellular localization of their interacting partners (Mogk et al. 2019).

\section{Cargo shuttle to reinforce the battlements}

Intracellular protozoan parasites have evolved unique machineries to export their proteins into extracellular compartments such as the parasitophorous vacuole (PV) or into host cytosol and across the host's cell membrane (Fig. 3). These parasites export $\sim 10 \%$ proteins as a survival strategy to acquire nutrients from host and remodel host cell membrane for immune evasion and for parasite egress (Przyborski and Lanzer 2004). The proteins exported into the PV follow the classical pathway (ER to Golgi-dependent) and non-classical pathway (Golgi-independent secretion) presumably through Golgi reassemblystacking protein (GRASP) (Struck et al. 2008). Though GRASP-mediated secretion is poorly explored in parasites, in other eukaryotes it is thought to mediate secretion of transmembrane proteins bypassing Golgi during cellular stress (Rabouille and Linstedt 2016).

The export of parasite proteins from the PV into the host cytoplasm requires specialized export machinery which is well studied mainly in Plasmodium (de Koning-Ward et al. 2016). Malarial parasites harbor a unique protein-conducting channel (PTEX machinery) which is absent in other parasites (de Koning-Ward et al. 2009). The proteins containing PEXEL or host-targeting motifs are exported through PTEX machinery into the host cell in a translocation-competent state (chaperone-protected, partially folded intermediate) (de Koning-Ward et al. 2009; Gehde et al. 2009; Mesén-Ramírez et al. 2016). One of the components of PTEX,
HSP101 (AAA+ ATPase), a hexameric unfoldase, helps in protein unfolding for its export across the translocon into the host cytoplasm (de Koning-Ward et al. 2009), where the polypeptide subsequently undergoes chaperone-assisted refolding. Thus, besides engaging its own chaperone machinery, the parasite may also utilize host chaperones for refolding its exported proteins. For instance, human HSP70 and HSP90 have also been found to be associated with malarial parasite protein HRP1. This parasite protein is involved in the formation of a knob-like structure on the infected RBC surface (Banumathy et al. 2002) which helps them to adhere to blood vessels and escape clearance from spleen.

Parasites also generate morphologically and functionally distinct transient secretory organelles such as J-dots, Maurer's cleft (Plasmodium), micronemes, rhoptries, dense granules (Plasmodium, Toxoplasma), and exosomes (Leishmania, Trypanosoma) (BayerSantos et al. 2013; Frénal et al. 2017; Silverman et al. 2010) (See Glossary). Some of these organelles harbor HSPs for correct targeting of exported parasite proteins (Bayer-Santos et al. 2013; Petersen et al. 2016; Silverman et al. 2010). Intriguingly, the HSP40 family (type I-IV based on J-domain organization) (Botha et al. 2007) is suggested to play a critical role in protein trafficking. For example, J-dot resident HSP70 and HSP40 (exported, type II) closely associate with $P$. falciparum virulence factor (PfEMP1) to facilitate its correct targeting to infected RBC membrane (Kulzer et al. 2010). Another exported knobassociated type II HSP40 (KAHSP40) is shown to interact with knob-forming genes like KAHRP and PfEMP1 (Acharya et al. 2012), which remodels infected RBCs, increases their cytoadherence, and prevents their clearance from spleen. Parasites have also acquired a unique class of essential exported type IV HSP40s (mainly studied in Plasmodium). Type IV HSP40 harbors a J-like domain, and its homolog is absent in human host. The divergent class of type IV HSP40s includes ring-infected erythrocyte surface antigen (RESA) protein which is released from dense granules into the host RBC during invasion. RESA binds to host spectrin to stabilize the RBC cytoskeleton and protect infected RBCs from thermal damage during febrile conditions (Pei et al. 2007).

During the initial stage of microbial infection, there is a large increase in the expression of host and microbial HSPs for their survival. However, through molecular mimicry, microbial HSPs induce a strong cross-reactive immune response against host HSPs leading to weakening of the host immune response (Giuliano et al. 2011; Henderson et al. 2006). 


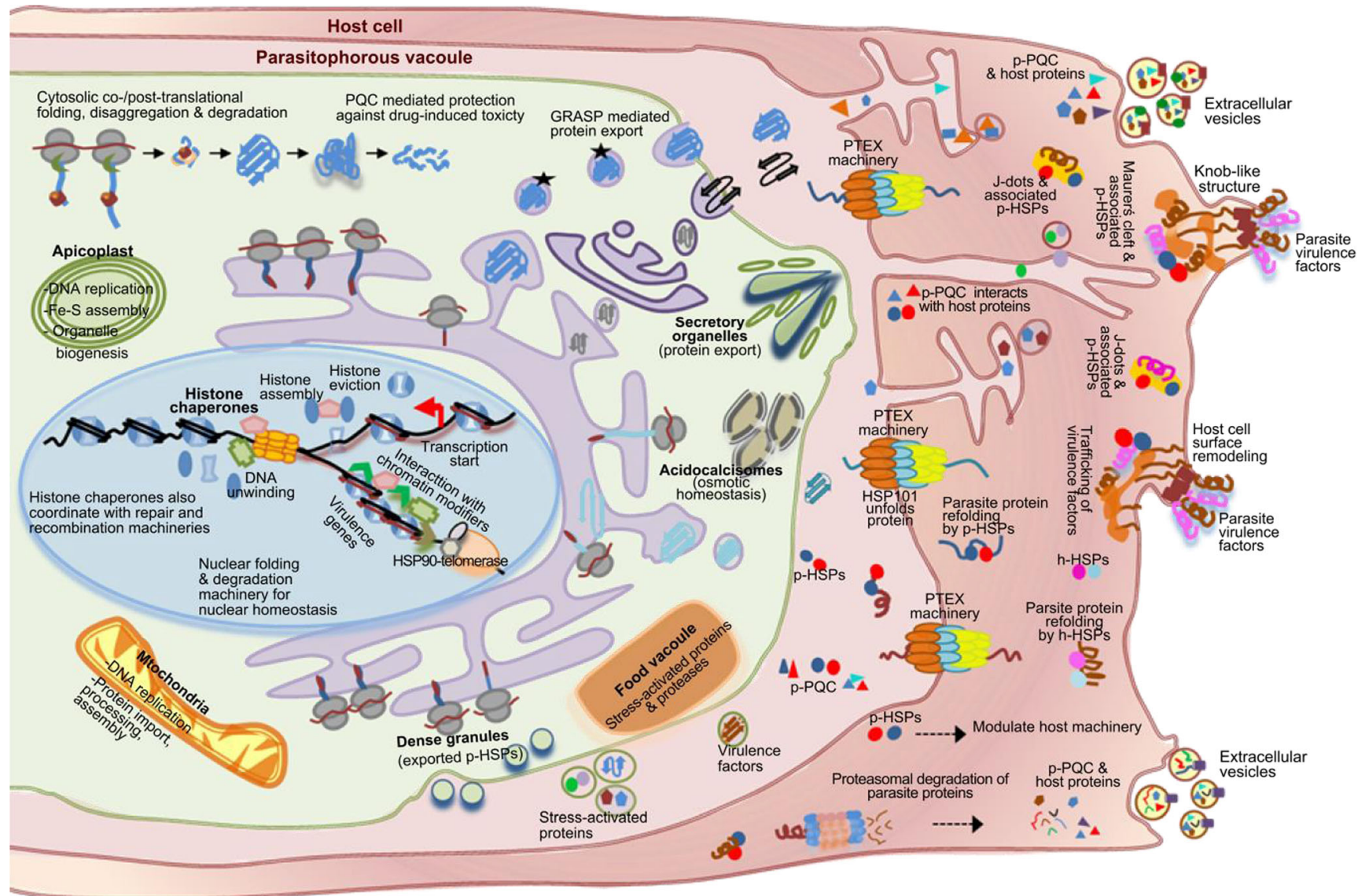

Fig. 3 Schematic representation of canonical and potential non-canonical roles of PQC machinery in Plasmodium falciparum (during asexual cycle in $\mathrm{RBC}$ ). The canonical roles include maintenance of cytosolic and organellar protein homeostasis by coordinating efficient folding/ assembly of nascent proteins and elimination of misfolded proteins, while the putative non-canonical roles include maintenance of nuclear and organellar genome dynamics, modulation of epigenetic machinery, organellar biogenesis, metabolic processes, buffering of drug-induced proteotoxicity, and export of critical proteins for parasite survival via classical and non-classical secretory mechanisms. These secretory processes may allow the secretion of stress-related enzymes, HSPs, and virulence factors into the parasitophorous vacuole (PV). The PTEX

Another strategy of immunomodulation involves influencing cytokine levels or complement activation. For instance, exported T. gondii HSP70 mediates inhibition of NF-KB which results in a decrease in host pro-inflammatory cytokines (Dobbin et al. 2002). Similarly, T. cruzi calreticulin is shown to inhibit the host complement system and this interaction is also thought to facilitate parasite invasion into the host cell. Calreticulin is an ER-resident chaperone but is also translocated to the surface of the parasite conventional secretion pathway. In fact, both host and parasite calreticulin is translocated to the membrane and released in the extracellular milieu (Ramírez et al. 2011). Based on literature from other microbes, it can be speculated that the parasite may also utilize machinery in the PV exports parasite proteins containing PEXEL or distinct targeting signals in an unfolded state into the host cytosol. The exported protein is refolded by the host HSPs (h-HSPs) and/or exported parasite HSPs (p-HSPs). In the host cytosol, the parasite generates transient organelles like Maurer's cleft and J-dots that are involved in trafficking of virulence factors to $\mathrm{RBC}$ surface for host cell remodeling. It can be hypothesized that (i) exported parasite-PQC (p-PQC) machinery may cross-talk with the host machineries for their own survival, and (ii) exported parasite cargo can be degraded by the host's proteasome into peptides which are released along with p-HSPs in extracellular vesicles (EVs). These EVs may modulate the host immune response or influence communication with other infected RBCs for gamete formation

the host surface-exposed HSP70, HSP60, HSP27, and calreticulin as adhesions to attach to the cell surface and to gain access to the intracellular milieu (Giuliano et al. 2011; Henderson et al. 2006). It can also be hypothesized that components of the parasite's ubiquitin-proteasome machinery exported into the host cytoplasm may influence host signaling and degradation of host proteins to support parasite intracellular growth (Loureiro and Ploegh 2006; Maculins et al. 2016). Further, the parasite also has to protect itself in the mosquito vector. Parasite PQC machinery may coordinate a defense against proteolytic attack by mosquito gut microbiota, melanization, ROS, and RNAi-mediated threats (Dubovskiy et al. 2013; Saraiva et al. 2016). 


\section{Buffering mutations and drug-induced proteotoxicity}

Various anthropogenic parameters (host/vector demography, climate, mass-drug administration programs) influence parasite evolution (genetic diversity, drug resistance) (Hoberg and Brooks 2015). For example, parasites adapt to drug pressure by acquiring spontaneous genetic mutations that may result in (i) reduced uptake or increased efflux of drug, (ii) affect protein-drug interaction, (iii) lead to metabolic bypass of the targeted pathway, and (iv) induce stress-response pathways (de Koning 2017). Studies in model organisms suggest that HSP90 serves as an evolutionary capacitor to compensate for the fitness cost associated with acquired mutations (Cowen and Lindquist 2005; Rutherford and Lindquist 1998). Parasite HSP90 may also buffer the acquired genetic mutations and protect the proteome from drug-induced proteotoxicity. In Leishmania, MAPK is essential for parasite growth and has been associated with antimony drug resistance. MAPK phosphorylates HSP70 and HSP90 in the foldosome complex to potentially regulate their stability and activity to buffer drug-induced proteotoxicity (Kaur et al. 2017). Similarly, sulfadiazine-resistant Toxoplasma gondii and benznidazole-resistant Trypanosoma cruzi clinical isolates exhibit differential expression of many HSPs in comparison to their respective drug-sensitive strains (Doliwa et al. 2013; Murta et al. 2008), while in Plasmodium falciparum, non-responsiveness against current frontline anti-malarial drug (artemisinin) has been associated with a mutation in kelch13, an adaptor protein of Cullin-E3 ligase. Mutation in PfKelch13 protein compromises the Cullin-E3 ligasemediated protein degradation activity, resulting in accumulation of PfPI3K and its lipid by-product phosphatidylinositol3-phosphate (PI3P), which is a key element in vesicular trafficking to different cellular parasite organelles. The parasite tries to recover from artemisinin-induced proteotoxic damage (protein alkylation) by activation of the unfolded protein response and upregulation of the TriC chaperone complex (Mok et al. 2015). Recently, it has been shown that the PfKelch13 mutant colocalizes in PI3P vesicles that are enriched with various $\mathrm{PQC}$ components, export machinery, and virulence factor (PfEMP1) (Haldar et al. 2018). These observations suggest that the parasite's PQC machinery balances fitness tradeoffs associated with acquired mutations that foster genetic diversity in parasite populations and enables natural selection of drug-resistant strains.

\section{Therapeutic challenges and hopes}

During parasitic infection, the host may remain aymptomatic and serve as a reservoir for parasite transmission or the host may develop a symptomatic infection. As the parasite burden increases, the host responds by an inflammatory attack followed by a cascade of immune reactions involving activation of the complement system, cell mediation, and antibody response (Melby et al. 2019). The host's immune surveillance is countered by parasite immune evasion strategies like parasite-mediated modification of the host complement system, altered antigen presentation, and induction of regulatory T-cells (Tregs) to dampen anti-parasitic response (Crompton et al. 2014; Dobbin et al. 2002; Dubey 2014; Ramírez et al. 2011). These evasion strategies also misdirect the host immune system towards self-antigens causing enormous immuno-pathological damage instead of protection against the parasite (Crompton et al. 2014). A major impediment in the treatment of parasitic infections is that in many cases the patient arrives at the health center only during acute infection, which becomes more difficult to control in pregnant mothers due to transplacental parasite transmission. Additionally, patients' non-compliance to drug regimens contributes to parasite recrudescence/relapse. Therefore, the challenges are (i) timely diagnosis and correct treatment to reduce parasite burden, (ii) restoration of host immunity to prevent secondary or concurrent infections, and (iii) follow-up parasite control programs post-treatment (anti-parasitic drug) to avoid recurrence of infection.

The existing anti-parasitic drugs inhibit protein targets critical for DNA replication, detoxification pathways, electron transport chain in mitochondria, and translation in cytosol/organelles. However, due to acquired point mutations in these parasitic drug targets, current therapies have become ineffective (Hefnawy et al. 2017; Phillips et al. 2017). Further, there is no effective vaccine available against intracellular protozoan parasites. Hence, there is an urgent need to identify robust alternative biological targets for rational drug design. The pleiotropic roles of components of PQC machinery make them attractive to explore for therapeutic targeting (Fig. 3). For instance, parasitic HSP90 is proposed as a potential anti-parasitic drug target because of its vital role in parasite invasion, replication, and stage differentiation. Small-molecule inhibitors (geldanamycin derivatives and other scaffolds) interfere with parasitic HSP90 activity and lead to parasite inhibition. But these small molecules also inhibit human HSP90 and exhibit only modest differences in binding affinities between host and parasite HSP90 (Wang et al. 2014). Similarly, exploring HSP60 and HSP70 as a vaccine candidate against parasitic infections has the drawback of crossrecognition of conserved epitopes and induction of autoimmune responses. Therefore, an effective approach would be to utilize multiple parasite antigens (especially diverged immunodominant antigens of PQC machinery) for evaluation of their prophylactic and therapeutic efficacy. For instance, the diverged HSP40 family (especially type IV class which is absent in humans) can be explored as potential targets for drug discovery and 
vaccine development (Daniyan and Blatch 2017). Further, their multistage expression may offer the additional advantage as a critical diagnostic biomarker for parasitic infections even at low parasite burden and for follow-up treatment in parasite control programs in geographically distinct populations.

Elaborate proof-of-concept studies are required to evaluate parasite HSPs as vaccine candidates or as adjuvants for delivery of parasite surface antigens to dendritic cells. However, due to geographical variability of parasitic infections, any vaccine candidate may face challenges: (i) variation in the immune response between the experimental model and the human population, (ii) differences due to genetic make-up and associated SNPs in host and parasite, (iii) idiosyncratic reactions in age or pregnancy-associated infections and in patients with HIV coinfections, and (iv) commercial viability of vaccine development. All these factors affect vaccine efficacy and limit the window of exploration of newer vaccine candidates. An alternative strategy would be to identify smallmolecule inhibitors for critical components of PQC machinery. But the existing lacunae in chemical therapeutic approaches are that most of these proteins have not been biochemically or structurally characterized and robust highthroughput drug screening platforms against these parasitic proteins/pathways are currently lacking. Many pharmaceutical companies are also hesitant to invest in drug discovery and development for parasitic infections due to the limited return on investment. Ongoing efforts include governmentsponsored anti-parasitic drug screening programs by academic institutes or a few public-private ventures (MMV, DNDi, Wellcome Trust, Gates Foundation, Consortium for Parasitic Drug Development) that are working towards the development of safe and efficacious next-generation medicines against drug-resistant parasitic infections. In addition to therapeutic approaches, intensive research efforts are required to strengthen our understanding of host-parasite-vector relationships and cooccurrence of infections and to develop innovative surveillance systems to track epidemiological patterns (Box 2).

Box 2 Some open questions in parasite research

\section{Concluding remarks}

With constant selection pressures from evolutionarily distant host and vector, intracellular parasites balance their survival, proliferation, and transmission. These critical decisions in the parasite's life cycle are influenced by dynamic components of PQC machinery. For a comprehensive understanding of the pleiotropic roles of the parasite's diverged PQC machinery, integrated efforts are required to delineate parasite pathways, immune evasion, and drug resistance mechanisms. These efforts will also help in setting robust drug-screening assays to determine the mode of action of potential anti-parasitic lead and the design of reliable invasive or non-invasive diagnosis for mixed infections. Overall, concerted initiatives will help in identifying alternative targets to develop cost-effective therapeutics and diagnosis strategies for point-of-care treatment regardless of geographical barriers.

Acknowledgments The authors thank the reviewers for their suggestions to improve the manuscript. The authors also thank Vidhi Mathur and Tani Agarwal for proof-reading the article. MA and VK acknowledge CSIR and UGC for fellowships. We apologize for not being able to cite work from various research groups due to restricted reference list. PlasmoDB, malaria parasite metabolic pathways, and TriTrypDB databases were used for data mining.

Funding information $\mathrm{AD}$ and $\mathrm{NG}$ are supported by INSA and DST-JCB research grants. NK is supported by INSA and DBT-IYBA research grants.

\section{Glossary}

Immunodominant

Protein antigens eliciting a strong immune response

Amastigote Non-motile intracellular stage of kinetoplastid present in the vertebrate host

Anthropogenic Changes in nature influenced by human activities

- How are parasite biological rhythms generated? Do they have endogenous time-keeping machinery which synchronizes with host/vector circadian rhythms? Or are parasites arrhythmic and exploit host/vector components to establish their biological rhythms for successful invasion, survival, nutrient management, and transmission?

- Does vector and host microbiota influence the reproductive and transmission rate of the parasite?

- Can protein quality control (PQC) machinery of intracellular parasites influence inter-species communication for progression of mixed infections (e.g., Leishmania \& M. tb or HIV; Plasmodium \& HIV; Plasmodium \& Trypanosoma), and co-endemicity of different parasitic infections and sharing of common vectors (e.g., malaria and filaria). Can the components of PQC machinery be explored as markers for a non-invasive (parasite antigens in urine) point-of-care diagnosis of mixed infections?

- Due to constant evolutionary arms race imposed by host/vector and drug-induced selection pressure, the parasite can develop mechanisms for numerous host/vector switching and refractory response to anti-parasitic drugs across different geographical locations. Are there appropriate global surveillance and disease forecasting programs which can help to devise preemptive measures for prevention of parasitic infections? What measures can be taken to minimize the evolutionary potential of parasites? 


Apicomplexan
Bradyzoites
Cytoplasmic stress
granules

Definitive host

Dense granules

\section{Exosomes}

Foldosome

Geographic mosaic

\section{GRASP}

Intermediate host

J-dots

Kinetoplastid
Spore-forming protozoan parasites harboring a relict plastid for metabolic processes and apical complex for host invasion Intracellular slowly multiplying encysted stage of Toxoplasma Membrane-less dynamic compartments containing stalled translation initiation complexes that accumulate upon stress.

These reservoirs allow the cell to rapidly respond to a changing environment

A primary host in which an infectious agent grows and develops into sexually matured stages Spherical secretory organelles in apicomplexans. Dense granule proteins are released soon after invasion, and they are associated with the PV membrane.

Extracellular vesicles containing protein, RNA, and lipid cargo.

A multi-chaperone complex comprising of HSP90 and HSP70 and their respective cochaperones

Interacting species that apply selection pressure on each other to coevolve in different geographical locations and communities.

Golgi reassembly stacking protein involved in Golgiindependent protein secretion

A secondary host in which the infectious agent multiplies asexually

Plasmodium-induced structures in infected RBCs and is involved in transport of virulence factors on the infected RBC surface for immune evasion

Flagellated protozoan parasites having unique mitochondrial DNA called kinetoplast DNA organized in a giant network of interlocked DNA
Maurer's cleft

Melanization

Metacyclic

Micronemes

Parasitophorous vacuole (PV)

PEXEL

Phenotypic plasticity

Pleiotropic roles

PQC

Plasmodium translocon of exported proteins (PTEX)

Promastigote

Rhoptries
Plasmodium-derived membranous structures in the cytoplasm of infected RBC. These are involved in sorting and export of parasite proteins on the RBC surface.

A defense mechanism adopted by insects to synthesize and deposit melanin around the pathogen.

Infective stage in the life cycle of parasite (Leishmania, Trypanosoma)

Ellipsoidal apical secretory organelles present in apicomplexans. Microneme proteins are involved in motility, invasion, and egress of the parasite.

Membrane-bound compartment derived from the host cell membrane which protects the parasite from host's cytopathic attacks

Plasmodium export element (Nterminus RxLxE/Q/D motif) used by $P$. falciparum to export parasite proteins into the cytoplasm of infected RBCs.

Ability to exhibit diverse phenotypes (biological roles) in different environmental conditions.

Heteromeric protein channel in parasitophorous vacuole membrane involved in transport of parasite proteins into the cytoplasm of infected RBCs Multiple biological roles protein quality control network comprising of folding, degradation, and processing machinery required for maintenance of cellular protein homeostasis

Flagellated extracellular stage of kinetoplastid with elongated morphology

Club-shaped apical secretory organelles in apicomplexans.

Rhoptry proteins are involved in invasion and intracellular survival of parasite. 
Sporozoite

Tachyzoites

Vector
Motile stage of spore-forming protozoan parasites which invades into the host cell to initiate the infection

Intracellular rapidly multiplying stage of Toxoplasma

An organism (vertebrate/ invertebrate) which transmits the infectious agent

\section{References}

Acharya P, Chaubey S, Grover M, Tatu U (2012) An exported heat shock protein 40 associates with pathogenesis-related knobs in Plasmodium falciparum infected erythrocytes. PLoS One 7:e44605

Aikawa M (2012) Intracellular parasitic protozoa (Elsevier)

Akopyants NS, Kimblin N, Secundino N, Patrick R, Peters N, Lawyer P, Dobson DE, Beverley SM, Sacks DL (2009) Demonstration of genetic exchange during cyclical development of Leishmania in the sand fly vector. Science (New York, NY) 324:265-268

Alsford S, Horn D (2012) Cell-cycle-regulated control of VSG expression site silencing by histones and histone chaperones ASF1A and CAF-1b in Trypanosoma brucei. Nucleic Acids Res 40:10150 10160

Alvira S, Cuellar J, Rohl A, Yamamoto S, Itoh H, Alfonso C, Rivas G, Buchner J, Valpuesta JM (2014) Structural characterization of the substrate transfer mechanism in Hsp70/Hsp90 folding machinery mediated by Hop. Nat Commun 5:5484

Bansal P, Tripathi A, Thakur V, Mohmmed A, Sharma P (2017) Autophagy-related protein ATG18 regulates apicoplast biogenesis in apicomplexan parasites. MBio 8:e01468-e01417

Banumathy G, Singh V, Tatu U (2002) Host chaperones are recruited in membrane-bound complexes by Plasmodium falciparum. J Biol Chem 277:3902-3912

Bates PA (2018) Revising Leishmania's life cycle. Nat Microbiol 3:529-530

Battistuzzi FU, Schneider KA, Spencer MK, Fisher D, Chaudhry S, Escalante AA (2016) Profiles of low complexity regions in Apicomplexa. BMC Evol Biol 16:47

Bayer-Santos E, Aguilar-Bonavides C, Rodrigues SP, Cordero EM, Marques AF, Varela-Ramirez A, Choi H, Yoshida N, da Silveira JF, Almeida IC (2013) Proteomic analysis of Trypanosoma cruzi secretome: characterization of two populations of extracellular vesicles and soluble proteins. J Proteome Res 12:883-897

Besteiro S, Williams RA, Morrison LS, Coombs GH, Mottram JC (2006) Endosome sorting and autophagy are essential for differentiation and virulence of Leishmania major. J Biol Chem 281:11384-11396

Bhartiya D, Chandramouli B, Kumar N (2015) Co-evolutionary analysis implies auxiliary functions of HSP110 in Plasmodium falciparum. Proteins 83:1513-1525

Botha M, Pesce E-R, Blatch GL (2007) The Hsp40 proteins of Plasmodium falciparum and other apicomplexa: regulating chaperone power in the parasite and the host. Int J Biochem Cell Biol 39: $1781-1803$

Burda PC, Caldelari R, Heussler VT (2017) Manipulation of the host cell membrane during plasmodium liver stage egress. mBio 8

Chaudhry SR, Lwin N, Phelan D, Escalante AA, Battistuzzi FU (2018) Comparative analysis of low complexity regions in Plasmodia. Sci Rep 8:335

Cortez C, Real F, Yoshida N (2016) Lysosome biogenesis/scattering increases host cell susceptibility to invasion by Trypanosoma cruzi metacyclic forms and resistance to tissue culture trypomastigotes. Cell Microbiol 18:748-760

Cowen LE, Lindquist S (2005) Hsp90 potentiates the rapid evolution of new traits: drug resistance in diverse fungi. Science 309:2185-2189

Craig EA, Marszalek J (2017) How do J-proteins get Hsp70 to do so many different things? Trends Biochem Sci 42:355-368

Crompton PD, Moebius J, Portugal S, Waisberg M, Hart G, Garver LS, Miller LH, Barillas-Mury C, Pierce SK (2014) Malaria immunity in man and mosquito: insights into unsolved mysteries of a deadly infectious disease. Annu Rev Immunol 32:157-187

Daniyan MO, Blatch GL (2017) Plasmodial Hsp40s: new avenues for antimalarial drug discovery. Curr Pharm Des 23:4555-4570

de Koning HP (2017) Drug resistance in protozoan parasites. Emerg Top Life Sci 1:627-632

de Koning-Ward TF, Gilson PR, Boddey JA, Rug M, Smith BJ, Papenfuss AT, Sanders PR, Lundie RJ, Maier AG, Cowman AF, Crabb BS (2009) A newly discovered protein export machine in malaria parasites. Nature 459:945-949

de Koning-Ward TF, Dixon MW, Tilley L, Gilson PR (2016) Plasmodium species: master renovators of their host cells. Nat Rev Microbiol 14: 494-507

DeZwaan DC, Freeman BC (2010) HSP90 manages the ends. Trends Biochem Sci 35:384-391

Dikic I (2017) Proteasomal and autophagic degradation systems. Annu Rev Biochem 86:193-224

Dobbin CA, Smith NC, Johnson AM (2002) Heat shock protein 70 is a potential virulence factor in murine Toxoplasma infection via immunomodulation of host NF- $\mathrm{kB}$ and nitric oxide. J Immunol 169:958-965

Doliwa C, Xia D, Escotte-Binet S, Newsham EL, Aubert D, Randle N, Wastling JM, Villena I (2013) Identification of differentially expressed proteins in sulfadiazine resistant and sensitive strains of Toxoplasma gondii using difference-gel electrophoresis (DIGE). Int J Parasitol Drugs Drug Resist 3:35-44

Dubey JP (2014) In: Gondii T (ed) The history and life cycle of Toxoplasma gondii, 2nd edn. Elsevier, pp 1-17

Dubovskiy I, Whitten M, Kryukov V, Yaroslavtseva O, Grizanova E, Greig C, Mukherjee K, Vilcinskas A, Mitkovets P, Glupov V (2013) More than a colour change: insect melanism, disease resistance and fecundity. Proc R Soc Lond B Biol Sci 280:20130584

Echeverria PC, Figueras MJ, Vogler M, Kriehuber T, de Miguel N, Deng B, Dalmasso MC, Matthews DE, Matrajt M, Haslbeck M, Buchner J, Angel SO (2010) The Hsp90 co-chaperone p23 of toxoplasma gondii: identification, functional analysis and dynamic interactome determination. Mol Biochem Parasitol 172:129-140

Ferguson HM, Read AF (2004) Mosquito appetite for blood is stimulated by Plasmodium chabaudi infections in themselves and their vertebrate hosts. Malar J 3:12

Flegr J, Prandota J, Sovičková M, Israili ZH (2014) Toxoplasmosis-a global threat. Correlation of latent toxoplasmosis with specific disease burden in a set of 88 countries. PLoS One 9:e90203

Frénal K, Dubremetz J-F, Lebrun M, Soldati-Favre D (2017) Gliding motility powers invasion and egress in Apicomplexa. Nat Rev Microbiol 15:645-660

Gehde N, Hinrichs C, Montilla I, Charpian S, Lingelbach K, Przyborski JM (2009) Protein unfolding is an essential requirement for transport across the parasitophorous vacuolar membrane of Plasmodium falciparum. Mol Microbiol 71:613-628

Giuliano JS Jr, Lahni PM, Wong HR, Wheeler DS (2011) Pediatric sepsis - part V: extracellular heat shock proteins: alarmins for the host immune system. Open Inflamm J 4:49-60 
Gupta MK, Agarawal M, Banu K, Reddy KS, Gaur D, Dhar SK (2018) Role of chromatin assembly factor 1 in DNA replication of Plasmodium falciparum. Biochem Biophys Res Commun 495: $1285-1291$

Haldar K, Bhattacharjee S, Safeukui I (2018) Drug resistance in Plasmodium. Nat Rev Microbiol 16:156-170

Hale VL, Watermeyer JM, Hackett F, Vizcay-Barrena G, van Ooij C, Thomas JA, Spink MC, Harkiolaki M, Duke E, Fleck RA, Blackman MJ, Saibil HR (2017) Parasitophorous vacuole poration precedes its rupture and rapid host erythrocyte cytoskeleton collapse in Plasmodium falciparum egress. Proc Natl Acad Sci U S A 114: 3439-3444

Hammond CM, Stromme CB, Huang H, Patel DJ, Groth A (2017) Histone chaperone networks shaping chromatin function. Nat Rev Mol Cell Biol 18:141-158

Hefnawy A, Berg M, Dujardin J-C, De Muylder G (2017) Exploiting knowledge on Leishmania drug resistance to support the quest for new drugs. Trends Parasitol 33:162-174

Henderson B, Allan E, Coates AR (2006) Stress wars: the direct role of host and bacterial molecular chaperones in bacterial infection. Infect Immun 74:3693-3706

Hoberg EP, Brooks DR (2015) Evolution in action: climate change, biodiversity dynamics and emerging infectious disease. Philos Trans R Soc Lond Ser B Biol Sci 370:20130553

Hombach A, Ommen G, MacDonald A, Clos J (2014) A small heat shock protein is essential for thermotolerance and intracellular survival of Leishmania donovani. J Cell Sci 127:4762-4773

Hübel A, Krobitsch S, Hörauf A, Clos J (1997) Leishmania major Hsp100 is required chiefly in the mammalian stage of the parasite. Mol Cell Biol 17:5987-5995

Kaur P, Garg M, Hombach-Barrigah A, Clos J, Goyal N (2017) MAPK1 of Leishmania donovani interacts and phosphorylates HSP70 and HSP90 subunits of foldosome complex. Sci Rep 7:10202

Kim DH, Hwang I (2013) Direct targeting of proteins from the cytosol to organelles: the ER versus endosymbiotic organelles. Traffic 14:613-621

Klaips CL, Jayaraj GG, Hartl FU (2018) Pathways of cellular proteostasis in aging and disease. J Cell Biol 217:51-63

Kulzer S, Rug M, Brinkmann K, Cannon P, Cowman A, Lingelbach K, Blatch GL, Maier AG, Przyborski JM (2010) Parasite-encoded Hsp40 proteins define novel mobile structures in the cytosol of the P. falciparum-infected erythrocyte. Cell Microbiol 12:1398-1420

Kumar A, Tanveer A, Biswas S, Ram EV, Gupta A, Kumar B, Habib S (2010) Nuclear-encoded DnaJ homologue of Plasmodium falciparum interacts with replication ori of the apicoplast genome. Mol Microbiol 75:942-956

LaCount DJ, Vignali M, Chettier R, Phansalkar A, Bell R, Hesselberth JR, Schoenfeld LW, Ota I, Sahasrabudhe S, Kurschner C et al (2005) A protein interaction network of the malaria parasite Plasmodium falciparum. Nature 438:103-107

Laurentino EC, Taylor S, Mair GR, Lasonder E, Bartfai R, Stunnenberg HG, Kroeze H, Ramesar J, Franke-Fayard B, Khan SM (2011) Experimentally controlled downregulation of the histone chaperone FACT in Plasmodium berghei reveals that it is critical to male gamete fertility. Cell Microbiol 13:1956-1974

Li F-J, He CY (2014) Acidocalcisome is required for autophagy in Trypanosoma brucei. Autophagy 10:1978-1988

Liévin-Le Moal V, Loiseau PM (2016) Leishmania hijacking of the macrophage intracellular compartments. FEBS J 283:598-607

Lodge R, Descoteaux A (2008) Leishmania invasion and phagosome biogenesis. In Molecular mechanisms of parasite invasion. Springer, pp. 174-181

Loureiro J, Ploegh HL (2006) Antigen presentation and the ubiquitinproteasome system in host-pathogen interactions. Adv Immunol 92:225-305
Lukes J, Basu S (2015) Fe/S protein biogenesis in trypanosomes - a review. Biochim Biophys Acta 1853:1481-1492

Maculins T, Fiskin E, Bhogaraju S, Dikic I (2016) Bacteria-host relationship: ubiquitin ligases as weapons of invasion. Cell Res 26:499-510

McCoy JM, Whitehead L, van Dooren GG, Tonkin CJ (2012) TgCDPK3 regulates calcium-dependent egress of Toxoplasma gondii from host cells. PLoS Pathog 8:e1003066

Melby PC, Stephens R, Dann SM (2019) Host defenses to protozoa. In: Clinical immunology, 5th edn. Elsevier, pp 425-435. e421

Mesén-Ramírez P, Reinsch F, Soares AB, Bergmann B, Ullrich A-K, Tenzer S, Spielmann T (2016) Stable translocation intermediates jam global protein export in Plasmodium falciparum parasites and link the PTEX component EXP2 with translocation activity. PLoS Pathog 12:e1005618

Messenger LA, Miles MA, Bern C (2015) Between a bug and a hard place: Trypanosoma cruzi genetic diversity and the clinical outcomes of Chagas disease. Expert Rev Anti-Infect Ther 13:995-1029

Miranda K, de Souza W, Plattner H, Hentschel J, Kawazoe U, Fang J, Moreno SN (2008) Acidocalcisomes in Apicomplexan parasites. Exp Parasitol 118:2-9

Mogk A, Ruger-Herreros C, Bukau B (2019) Cellular functions and mechanisms of action of small heat shock proteins. Annu Rev Microbiol 73

Mok S, Ashley EA, Ferreira PE, Zhu L, Lin Z, Yeo T, Chotivanich K, Imwong M, Pukrittayakamee S, Dhorda M (2015) Population transcriptomics of human malaria parasites reveals the mechanism of artemisinin resistance. Science (New York, NY) 347:431-435

Montagna GN, Buscaglia CA, Munter S, Goosmann C, Frischknecht F, Brinkmann V, Matuschewski K (2012) Critical role for heat shock protein 20 (HSP20) in migration of malarial sporozoites. J Biol Chem 287:2410-2422

Morales MA, Watanabe R, Dacher M, Chafey P, Fortéa JO, Scott DA, Beverley SM, Ommen G, Clos J, Hem S (2010) Phosphoproteome dynamics reveal heat-shock protein complexes specific to the Leishmania donovani infectious stage. Proc Natl Acad Sci 107: 8381-8386

Mott A, Lenormand G, Costales J, Fredberg JJ, Burleigh BA (2009) Modulation of host cell mechanics by Trypanosoma cruzi. J Cell Physiol 218:315-322

Muralidharan V, Goldberg DE (2013) Asparagine repeats in Plasmodium falciparum proteins: good for nothing? PLoS Pathog 9:e1003488

Murta SM, Nogueira FB, dos Santos PF, Campos FM, Volpe C, Liarte DB, Nirdé P, Probst CM, Krieger MA, Goldenberg S (2008) Differential gene expression in Trypanosoma cruzi populations susceptible and resistant to benznidazole. Acta Trop 107:59-65

Navadgi VM, Chandra BR, Mishra PC, Sharma A (2006) The two Plasmodium falciparum nucleosome assembly proteins play distinct roles in histone transport and chromatin assembly. J Biol Chem 281: 16978-16984

Ocaña-Mayorga S, Llewellyn MS, Costales JA, Miles MA, Grijalva MJ (2010) Sex, subdivision, and domestic dispersal of Trypanosoma cruzi lineage I in southern Ecuador. PLoS Negl Trop Dis 4:e915

O'Shea B, Rebollar-Tellez E, Ward R, Hamilton J, El Naiem D, Polwart A (2002) Enhanced sandfly attraction to Leishmania-infected hosts. Trans R Soc Trop Med Hyg 96:117-118

Pallavi R, Roy N, Nageshan RK, Talukdar P, Pavithra SR, Reddy R, Venketesh S, Kumar R, Gupta AK, Singh RK, Yadav SC, Tatu U (2010) Heat shock protein 90 as a drug target against protozoan infections: biochemical characterization of HSP90 from Plasmodium falciparum and Trypanosoma evansi and evaluation of its inhibitor as a candidate drug. J Biol Chem 285:37964-37975

Pavithra SR, Kumar R, Tatu U (2007) Systems analysis of chaperone networks in the malarial parasite Plasmodium falciparum. PLoS Comput Biol 3:e168-e1715 
Pei X, Guo X, Coppel R, Bhattacharjee S, Haldar K, Gratzer W, Mohandas N, An X (2007) The ring-infected erythrocyte surface antigen (RESA) of Plasmodium falciparum stabilizes spectrin tetramers and suppresses further invasion. Blood 110:1036-1042

Perez-Morales D, Espinoza B (2015) The role of small heat shock proteins in parasites. Cell Stress Chaperones 20:767-780

Pesce E-R, Maier AG, Blatch GL (2014) Role of the Hsp40 family of proteins in the survival and pathogenesis of the malaria parasite. In: Heat shock proteins of malaria. Springer, pp 71-85

Petersen W, Kulzer S, Engels S, Zhang Q, Ingmundson A, Rug M, Maier AG, Przyborski JM (2016) J-dot targeting of an exported HSP40 in Plasmodium falciparum-infected erythrocytes. Int J Parasitol 46: 519-525

Phillips MA, Burrows JN, Manyando C, van Huijsduijnen RH, Van Voorhis WC, Wells TNC (2017) Malaria. Nat Rev Dis Primers 3: 17050

Piña-Vázquez C, Reyes-López M, Ortíz-Estrada G, De la Garza M, Serrano-Luna J (2012) Host-parasite interaction: parasite-derived and-induced proteases that degrade human extracellular matrix. J Parasitol Res 2012:1-24

Powers ET, Balch WE (2013) Diversity in the origins of proteostasis networks - a driver for protein function in evolution. Nat Rev Mol Cell Biol 14:237-248

Przyborski J, Lanzer M (2004) The malarial secretome. Science (New York, NY) 306:1897-1898

Rabouille C, Linstedt AD (2016) GRASP: a multitasking tether. Front Cell Dev Biol 4:1

Ramírez G, Valck C, Ferreira VP, López N, Ferreira A (2011) Extracellular Trypanosoma cruzi calreticulin in the host-parasite interplay. Trends Parasitol 27:115-122

Real F, Florentino PT, Reis LC, Ramos-Sanchez EM, Veras PS, Goto H, Mortara RA (2014) Cell-to-cell transfer of Leishmania amazonensis amastigotes is mediated by immunomodulatory LAMP-rich parasitophorous extrusions. Cell Microbiol 16:1549-1564

Requena JM, Montalvo AM, Fraga J (2015) Molecular chaperones of Leishmania: central players in many stress-related and -unrelated physiological processes. Biomed Res Int 2015:301326

Rutherford SL, Lindquist S (1998) Hsp90 as a capacitor for morphological evolution. Nature 396:336-342

Ryan U, Fayer R, Xiao L (2014) Cryptosporidium species in humans and animals: current understanding and research needs. Parasitology 141:1667-1685

Salcedo-Sora JE, Caamano-Gutierrez E, Ward SA, Biagini GA (2014) The proliferating cell hypothesis: a metabolic framework for Plasmodium growth and development. Trends Parasitol 30:170-175

Saraiva RG, Kang S, Simões ML, Angleró-Rodríguez YI, Dimopoulos G (2016) Mosquito gut antiparasitic and antiviral immunity. Dev Comp Immunol 64:53-64

Sawarkar R, Paro R (2013) Hsp90@ chromatin. Nucleus: an emerging hub of a networker. Trends Cell Biol 23:193-201

Scher R, Garcia JBF, Pascoalino B, Schenkman S, Cruz AK (2012) Characterization of anti-silencing factor 1 in Leishmania major. Mem Inst Oswaldo Cruz 107:377-386

Schmidt JC, Manhaes L, Fragoso SP, Pavoni DP, Krieger MA (2018) Involvement of STI1 protein in the differentiation process of Trypanosoma cruzi. Parasitol Int 67:131-139

Schopf FH, Biebl MM, Buchner J (2017) The HSP90 chaperone machinery. Nat Rev Mol Cell Biol 18:345-360

Shonhai A, Maier AG, Przyborski JM, Blatch GL (2011) Intracellular protozoan parasites of humans: the role of molecular chaperones in development and pathogenesis. Protein Pept Lett 18:143-157
Silverman JM, Clos J, de'Oliveira CC, Shirvani O, Fang Y, Wang C, Foster LJ, Reiner NE (2010) An exosome-based secretion pathway is responsible for protein export from Leishmania and communication with macrophages. J Cell Sci 123:842-852

Singh GP, Chandra BR, Bhattacharya A, Akhouri RR, Singh SK, Sharma A (2004) Hyper-expansion of asparagines correlates with an abundance of proteins with prion-like domains in Plasmodium falciparum. Mol Biochem Parasitol 137:307-319

Spielmann T, Montagna GN, Hecht L, Matuschewski K (2012) Molecular make-up of the Plasmodium parasitophorous vacuolar membrane. Int J Med Microbiol 302:179-186

Struck NS, Herrmann S, Langer C, Krueger A, Foth BJ, Engelberg K, Cabrera AL, Haase S, Treeck M, Marti M, Cowman AF, Spielmann T, Gilberger TW (2008) Plasmodium falciparum possesses two GRASP proteins that are differentially targeted to the Golgi complex via a higher- and lower-eukaryote-like mechanism. J Cell Sci 121: 2123-2129

Szoor B, Haanstra JR, Gualdron-Lopez M, Michels PA (2014) Evolution, dynamics and specialized functions of glycosomes in metabolism and development of trypanosomatids. Curr Opin Microbiol 22:79-87

Teixeira F, Tse E, Castro H, Makepeace KA, Meinen BA, Borchers CH, Poole LB, Bardwell JC, Tomás AM, Southworth DR (2019) Chaperone activation and client binding of a 2-cysteine peroxiredoxin. Nat Commun 10:659

Týč J, Klingbeil MM, Lukeš J (2015) Mitochondrial heat shock protein machinery hsp70/hsp40 is indispensable for proper mitochondrial DNA maintenance and replication. mBio 6:e2425-e02414

Vonlaufen N, Kanzok SM, Wek RC, Sullivan WJ Jr (2008) Stress response pathways in protozoan parasites. Cell Microbiol 10: $2387-2399$

Voth W, Jakob U (2017) Stress-activated chaperones: a first line of defense. Trends Biochem Sci 42:899-913

Vyas A (2015) Mechanisms of host behavioral change in toxoplasma gondii rodent association. PLoS Pathog 11:e1004935

Walczak M, Ganesan SM, Niles JC, Yeh E (2018) Atg8 is essential specifically for an autophagy-independent function in apicoplast biogenesis in blood-stage malaria parasites. MBio 9:e02021e02017

Walker DM, Oghumu S, Gupta G, McGwire BS, Drew ME, Satoskar AR (2014) Mechanisms of cellular invasion by intracellular parasites. Cell Mol Life Sci 71:1245-1263

Walochnik J, Auer H, Joachim A (2017) Parasitic infections in humans and animals. In: Comparative Medicine. Springer, pp 177-189

Wang T, Bisson WH, Mäser P, Scapozza L, Picard D (2014) Differences in conformational dynamics between Plasmodium falciparum and human Hsp90 orthologues enable the structure-based discovery of pathogen-selective inhibitors. J Med Chem 57:2524-2535

WHO (2014) A global brief on vector-borne diseases (World Health Organization)

Yebenes H, Mesa P, Munoz IG, Montoya G, Valpuesta JM (2011) Chaperonins: two rings for folding. Trends Biochem Sci 36: 424-432

Publisher's note Springer Nature remains neutral with regard to jurisdictional claims in published maps and institutional affiliations. 